\title{
Study of an Optimum Design Method for Links
}

\author{
Shujun $\mathrm{Hu}^{1, *}$ and Zhan Wang ${ }^{2}$ \\ ${ }^{1}$ School of Civil Engineering and Architecture, Nanchang University, Nanchang 330031, China \\ ${ }^{2}$ State Key Laboratory of Subtropical Building Science, South China University of Technology, Guangzhou 510640, \\ China
}

\begin{abstract}
Under severe earthquakes, eccentrically braced frames might experience large inelastic deformations, and the inelastic action is restricted primarily to the ductile links. In order to study the reasonable design method for links, seventy analyses of links are conducted to investigate the effect of different flange width-thickness ratio and length, fifty links are also designed to study the influence of stiffeners spacing, stiffeners thickness and placing on side(s), and thirty-six links are designed to consider the effect of axial loads, which are all based on the material properties of Q235 steel. The accuracy of finite element models is verified using the experimental data during cyclic loading. Numerical analysis results show that the flange width-thickness ratio of short and long links can be relaxed to $10 \sqrt{235 / f y}$, and stiffeners can only be placed on one side. However, the flange width-thickness ratio of intermediate links is limited to $8 \sqrt{235 / \mathrm{fy}}$, and stiffeners should be placed on both sides due to the unstable behavior. Stiffener thickness has no significant influence on the performance of links with varying length. Unlike short links, intermediate and long links are susceptible to the axial forces. Then an optimum design method is proposed by analyzing the main influencing factors, so links can have good ductility and stiffness at high load levels.
\end{abstract}

Keywords: Eccentrically braced frames (EBFs), finite element method, flange width-thickness ratio, link, stiffener.

\section{INTRODUCTION}

Eccentrically braced frames (EBFs), which combined the advantages of moment resisting frames (MRFs) and concentrically braced frames (CBFs), can exhibit both adequate ductility and lateral stiffness during rare earthquake. The inelastic deformations were only limited to links while the other members, including columns, beam segment outside of links and columns, were designed to remain essentially elastic, so damage on the EBFs can be controlled. Moreover, the ductility mainly depended on links, which was determined by the inelastic rotational capacity and energy dissipation [1-5]. Therefore, links were the most important members in EBFs, and the seismic performance of EBFs can be improved by reasonable design of the links.

Link length has a great influence on the inelastic rotation $\gamma_{p}$. The length ratio $\rho$ is equal to $e V_{p} / M_{p}$, where $e$ is the link length, $M_{p}$ and $V_{p}$ are the nominal plastic flexural and shear strength, respectively. According to AISC [6], Links with $\rho$ less than 1.6, called short(shear) links, form plastic hinges at either end and $\gamma_{p}$ can be designed for 0.08 rad. Links with $\rho$ more than 2.6, called long (flexural) links, yield primarily in flexural and $\gamma_{p}$ can be designed for 0.02 rad. Links with $\rho$ between 1.6 and 2.6, called intermediate (shear-flexural) links, yield combined of shear and flexural, and $\gamma_{p}$ can be designed by linear interpolation between 0.08 and 0.02

*Address correspondence to this author at the School of Civil Engineering and Architecture, Nanchang University, Nanchang 330031, China; Tel:(+86)15579183858; Fax: (+86)15579183421 , (+83)2087113421; E-mail:husj.1229@163.com depending on the length ratio $\rho$. Many designers preferred to use links yielded in shear because they showed better stiffness, strength and ductility. However, architectural consideration restricted the application of short links in some situations, so intermediate and long links were also important and inevitable to be used.

As the high significance of the links in the overall performance of the EBFs, many researchers had been dedicated to study the seismic behaviour of links, including the effect of flange with-thickness ratio [7], loading protocol [8], stiffeners [9], overstrength factor [10], tubular links [11] and the performance of intermediate and long links [12]. However, more investigations were concentrating on the independence parameters or part of links. In addition, previous studies mainly focused on the A36 [1] , A992 [9] and European steel [13], and the performance of links with different steel is quite different. So it is not known whether the parameters and design method of links given by the AISC can be used for the links with Q235 or Q345 steel. Based on the Q235 steel, a comprehensive analysis of all the links with varying length ratio should be studied, and the maximum inelastic rotation and energy dissipation can be obtained. The influencing factors of links mainly include flange width-thickness ratio, stiffener thickness and placing on side(s), stiffener spacing and axial forces. Moreover, the current flange width-thickness requirement for all the links was derived from Code for seismic design of buildings [14], it may be conservatively for some links, so many rolled steel cross-sections cannot be used as links, and it is also 
necessary to increase the sizes of links to satisfy the value.

In this study, finite element analysis method is used to consider the main influencing factors of links, and the finite element modeling is verified by the existing experimental data, so numerical analysis of links with length varying from short links to long links can be studied. The width- thickness ratio is reevaluated by modeling seventy links, and the reasonable arrangements of stiffeners spacing, stiffeners thickness and placing on side(s) are also investigated by analyzing fifty links. The performance of links under axial forces is considered by thirty-six analysis models. According to the analysis result, an optimum design method for all types of links with I-shaped cross-section can be obtained, so it can provide the designers with suggestions to ensure a better and safe scheme of these links.

\section{FINITE ELEMENT MODELING}

The accuracy of finite element model of links was investigated, and it was developed to predict the performance of specimen 7 and 9 tested by Okazaki [9]. The hysteretic curves, failure modes, maximum inelastic rotations are compared. Finite element analyses are carried out with same loading protocol, boundary conditions and material properties by using ANSYS.

\subsection{Modeling Approach}

Many solid and shell elements can be used to model links in ANSYS, and solid 45 is chosen to the most proper element [15]. This element is defined by eight nodes and each end node having three degrees of freedom is suitable for nonlinear large displacement. Plasticity, creep, stress stiffening, large deflection and large strain can be considered in this element. Since the local instability, residual stress and low cycle fatigue effect of links are not considered, models can be meshed uniformly without local refinement in the high stress regions, and only one element is employed in the thickness of flange and web [12].

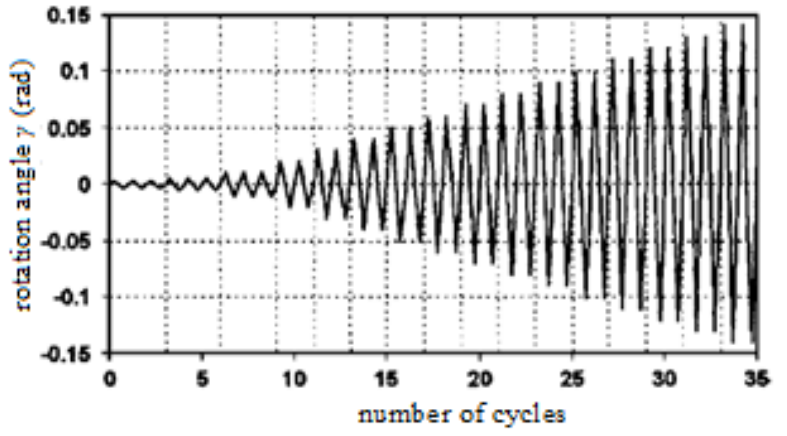

Fig.(1). Loading protocol.

The performance of model links is also influenced by the boundary conditions. Rotations at nodes on both links ends are all constrained, and left end nodes are permitted to horizontal translation but they are restrained to vertical translation, right nodes are restrained to horizontal translation and loads are applied by imposing vertical displacement. In this manner, shear forces and equal ends moments with no axial force can load on the links [7].

AISC loading protocol [6] for links is used in the finite element analysis, which is the same as the experimental research, as shown in Fig. (1). The loading protocol controls the link rotation angle, $\gamma$, which is computed as the relative displacement applied at right end of the link.

\subsection{Model Validation}

For the specimen 7 and 9 created to validate the finite element models, material properties of ASTM A992 steel are considered as the test results [9].

\subsubsection{Specimen 7 - Long Link}

Specimen 7 is a long link and the length ratio $\rho$ is 3.29. The stiffeners only place on one side and have a distance of $305 \mathrm{~mm}$ from each end. Fig. (2) compares the failure modes
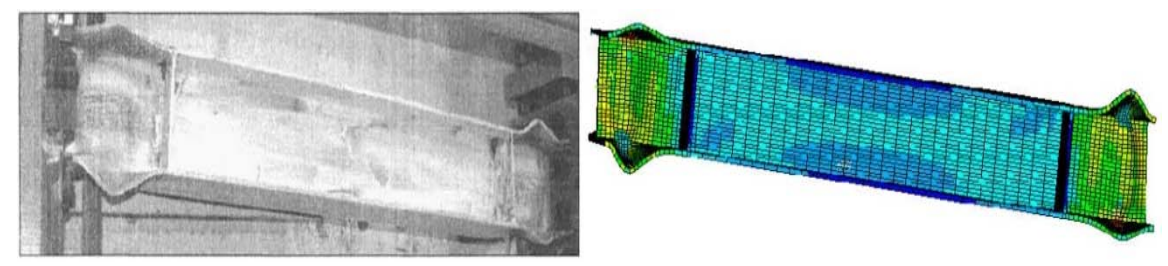

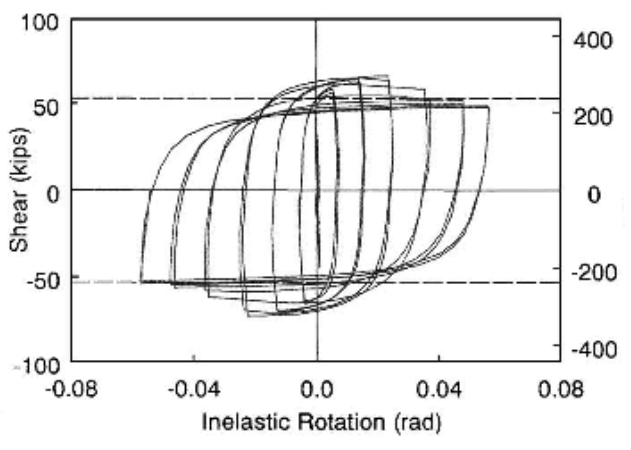

(a) Experimental research

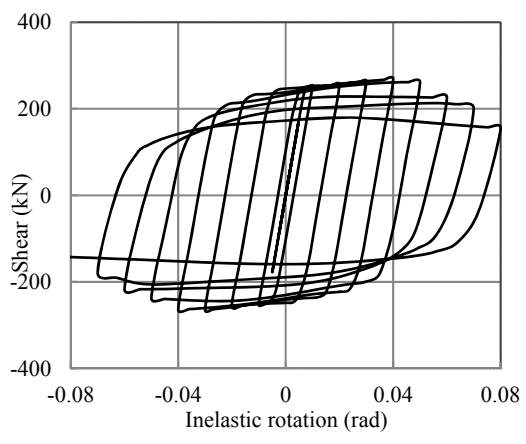

(b) Finite element model

Fig. (2). Comparison of deformed and shear versus inelastic rotation hysteresis for specimen 7. 
and hysteresis curves of the experimental testing and finite element analysis. Both of link failure modes are flange and web local buckling followed by fracture in web. The maximum inelastic rotations of experimental and FE model are 0.037 and 0.045 respectively, which are comparable and greater than the specified by the provision. The maximum shear forces and hysteresis curves are also close conformance.

\subsubsection{Specimen 9 - Intermediate Link}

Specimen 9 is an intermediate link and the length ratio $\rho$ is 1.95 . The stiffeners only place on one side and have a distance of 5@203. The failure modes and hysteresis curves of the experimental research and finite element analysis are also compared, as shown in Fig. (3). The failure modes of both models are flange and web local buckling. The maximum inelastic rotations of experimental and finite element model are 0.044 and 0.048 respectively, but they cannot reach the specified by the provision, it may be caused by the reduced strength due to the premature failure of flange and web. The maximum shear forces and hysteresis curves are also close conformance.

Based on reasonably predict the rotation, the proposed finite element modeling can be used in the analysis of links.

\section{PARAMETRIC STUDY FOR LINKS}

The influencing factors of links mainly include flange width-thickness ratio, stiffener thickness and placing on side(s), stiffener spacing. In addition, the effect of axial forces for links with varying length ratio cannot be ignored. So many types of links models are designed and analyzed which are all based on Q235 steel.

\subsection{Material Property}

For modeling material property of Q235 steel, multilinear kinematic hardening curve including softening segment with von mises yielding criterion is applied during cyclic loading, and the multilinear stress-strain curve [16] is shown in Fig. (4). A modulus of elasticity is $206000 \mathrm{~N} / \mathrm{mm}^{2}$ and Poisson's ratio is 0.3 , the yield stress $f_{y}$, ultimate stress $f_{u}$ and softening segment stress $f_{t}$ are 235,420 and $330 \mathrm{~N} / \mathrm{mm}^{2}$, respectively. The yield strain $\varepsilon_{y}$, ultimate strain $\varepsilon_{u}$ and descend segment strain $\varepsilon_{t}$ are $0.114 \%, 15 \%$ and $22 \%$ corresponding to these stresses.

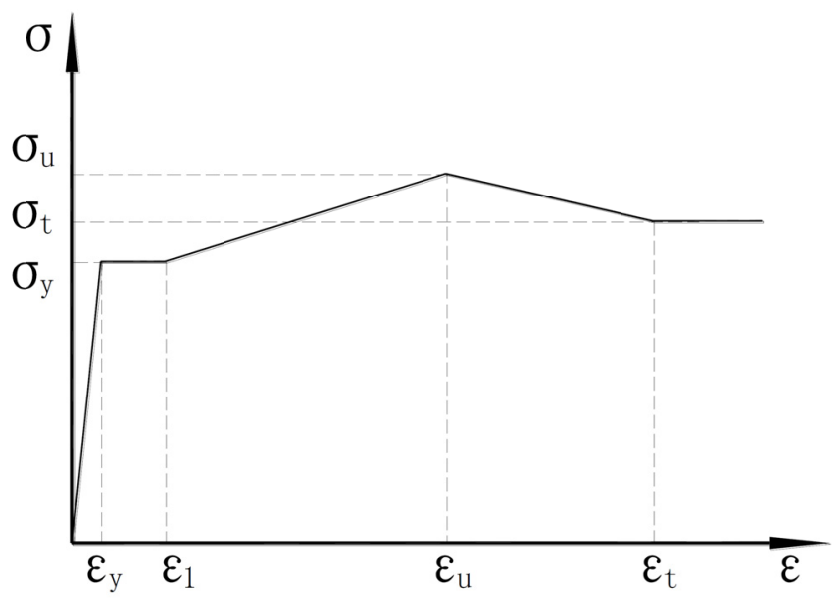

Fig.(4). Multilinear stress-strain curve of Q235 steel.

\subsection{Flange Width-Thickness Ratio}

The current maximum flange width-thickness ratio of links is limited to $8 \sqrt{235 / f_{\mathrm{y}}}$ by the Code for seismic design of buildings [14]. The value is mainly derived from AISC which are based on A36 or A992 steel. It may be conservative for some types of links with Q235 steel. So seventy links with varying length ratio are designed and analyzed to reevaluate the flange width-thickness ratio.

Seventy links models including short, intermediate and long links, based on $\mathrm{H} 400 \times 250 \times 10 \times b_{f}$ and changed the flange width $b_{f}$ and length $l$, are designed to investigate the effect of flange width-thickness ratio. The stiffeners are only
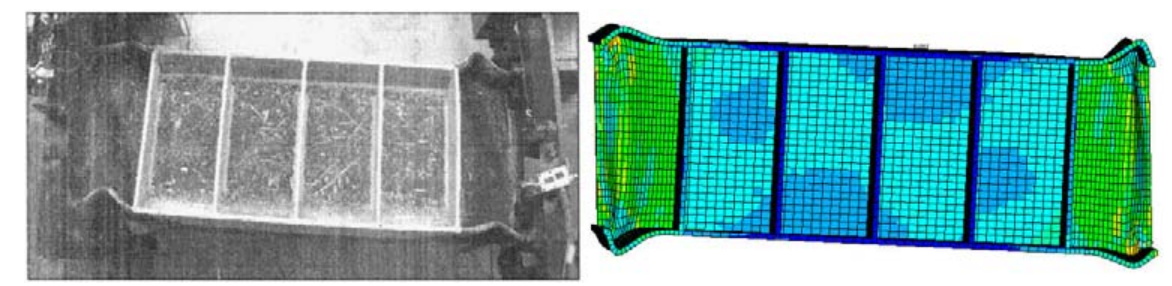

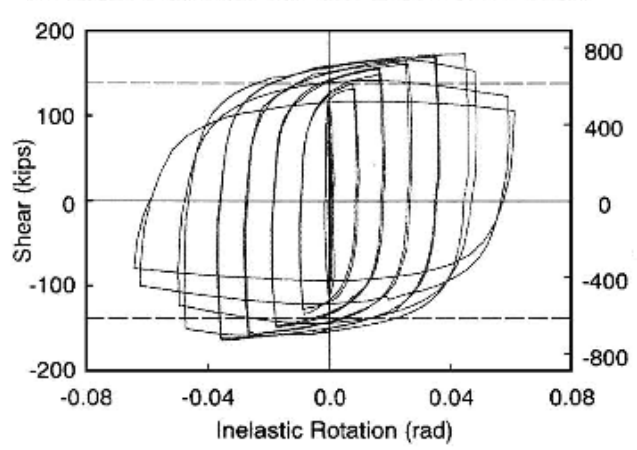

(a) Experimental research

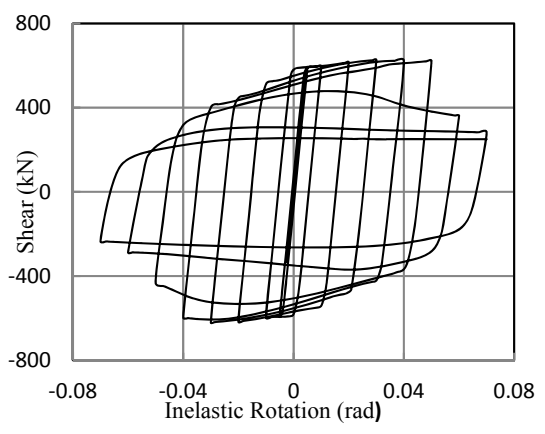

(b) Finite element model

Fig. (3). Comparison of deformed and shear versus inelastic rotation hysteresis for specimen 9 . 
placed on one side because the section depth is less than $640 \mathrm{~mm}$. The stiffener thickness, stiffener spacing and web slenderness ratio can all satisfy the requirements of current specification [14].

The maximum inelastic rotation $\gamma_{\max }$ that a link achieves prior to strength degradation is determined from a backbone curve by extracting the points at the maximum displacement of each analysis step in the hysteresis curve. If strength degradation is not happened in the backbone curve, the maximum applied inelastic rotation is chosen as the plastic rotation capacity [12].

Fig. (5) shows the maximum inelastic rotation $\gamma_{\max }$ versus length ratio $\rho$, and Fig. (6) shows the normalized inelastic rotation (maximum inelastic rotation/design rotation) versus length ratio for all of seventy links. Short links, intermediate links and long links are indicated by squares, triangles and rhomboids. All of the filled icons indicate links satisfy the requirement of flange width-flange ratio $\left(b_{f} / t_{f}<8 \sqrt{235 / f_{\mathrm{y}}}\right)$, and the empty icons are those that exceed the value. A solid line indicates the allowable maximum design rotation. From Fig.(5) and Fig. (6), all of the short links that include filled and empty icons can reach the maximum design rotation, the intermediate links with filled rhomboids can also satisfy the requirement, but some intermediate links with empty rhomboids cannot reach the design rotation because of the premature failure of flange and web.

Fig.(7) shows the normalized inelastic rotation versus flange width-thickness ratio for all of the link models. In Fig. (6), all of links satisfy the current flange width-thickness ratio $8 \sqrt{235 / f_{\mathrm{y}}}$ can reach the design rotation. As the flange width-thickness ratio is relaxed to $10 \sqrt{235 / f}$, all of the short and long links can reach the requirement of design rotation, but some intermediate cannot reach the design rotation due to the premature failure. These results indicate that the relaxation of flange width-thickness ratio to $10 \sqrt{235 / f}$ y can be justified for short and long links, and the value of intermediate links is also limited to $8 \sqrt{235 / \text { fy }}$.

\subsection{Stiffener Spacing and Placing}

In order to investigate the influences of stiffener spacing and placing, links with varying length from short links to

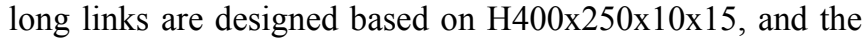
section properties, stiffener spacing and stiffener spacing are all shown in Table $\mathbf{1}$.

Short links. The length and length ratio $\rho$ of all the short links are $1200 \mathrm{~mm}$ and 1.44 , respectively, and the design

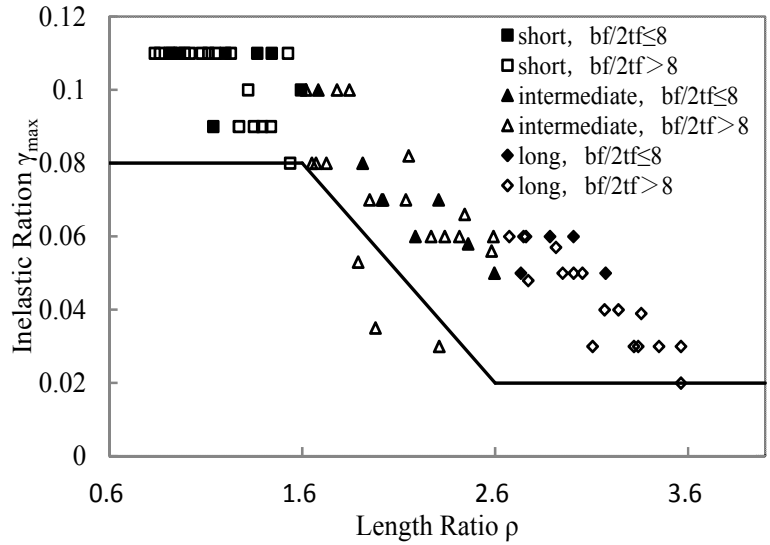

Fig.(5). Inelastic rotation versus length ratio for links

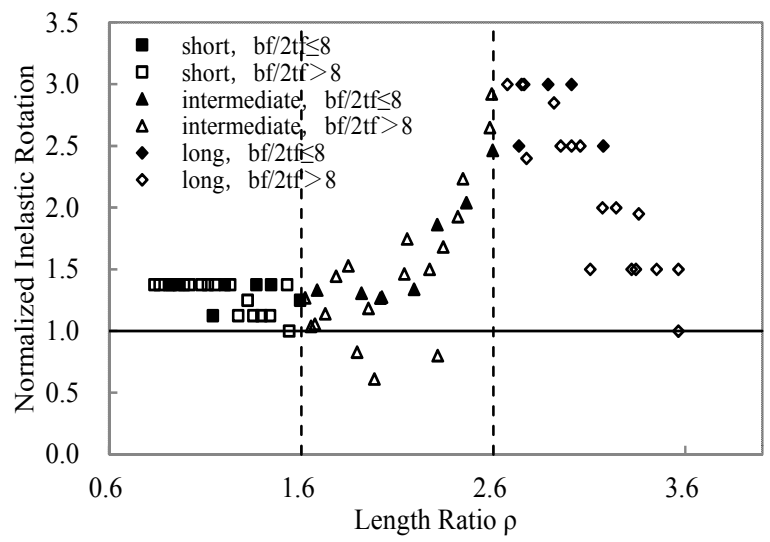

Fig. (6). Normalized rotation versus length ratio for links.

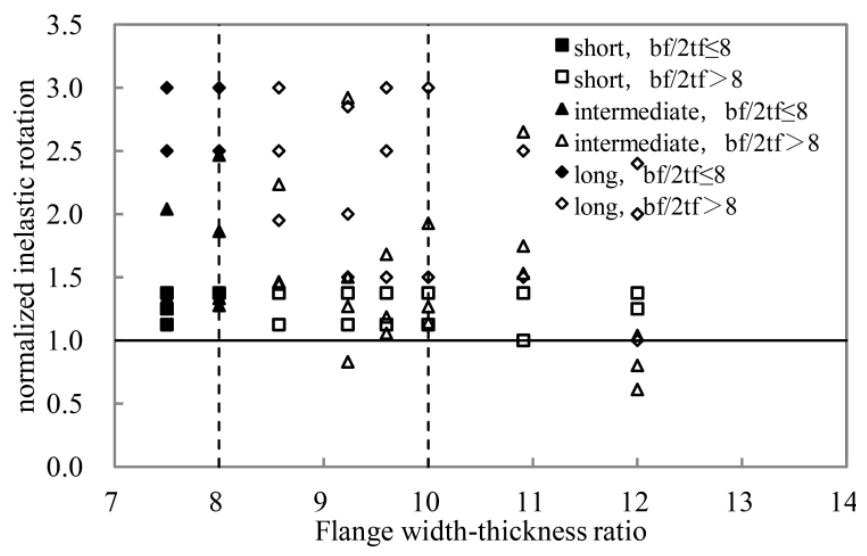

Fig. (7). Normalized rotation versus flange width-thickness ratio for links.

Table 1. Section properties for links with varying stiffener spacing and placing

\begin{tabular}{|c|c|c|c|c|c|}
\hline Section & Type & Length & Length ratio, $\rho$ & Stiffener spacing & Stiffener stiffness \\
\hline \hline $400 \times 250 \times 10 \times 15$ & short & $1200 \mathrm{~mm}$ & 1.44 & $4 @ 300,5 @ 240,6 @ 200,7 @ 171,8 @ 150$ & $\mathrm{t}_{\mathrm{a}}=10 \mathrm{~mm}$ \\
\hline $400 \times 250 \times 10 \times 15$ & intermediate & $1680 \mathrm{~mm}$ & 2.02 & $4 @ 420,5 @ 336,6 @ 280,7 @ 240,8 @ 210$ & $\mathrm{t}_{\mathrm{a}}=10 \mathrm{~mm}$ \\
\hline $400 \times 250 \times 10 \times 15$ & long & $2400 \mathrm{~mm}$ & 2.88 & $\begin{array}{c}5 @ 480,6 @ 400,7 @ 342,8 @ 300,9 @ 266, \\
10 @ 240, \text { a distance of 305mm from each end }\end{array}$ \\
\hline
\end{tabular}


rotation is 0.08 . From Fig. (8a), the inelastic rotations of links with tighter stiffeners spacing can increase to 0.11 , and inelastic rotations of links with the maximum allowable spacing are close to the required by the provision. However, it can't reach the design rotation as the stiffener spacing is larger than the required value. The stiffener places one or two sides will have no significant effect on the inelastic rotation and hysteresis curve of links with allowable stiffener spacing, as shown in Fig. (9a). So the stiffener spacing of short links should only be less than the required spacing by the provision, and stiffeners placing one or two sides will have good agreement.

Intermediate links. The length and length ratio $\rho$ of all the intermediate links are $1680 \mathrm{~mm}$ and 2.02 , respectively, and the design rotation is 0.055 . From Fig. (8b), the effects of stiffeners spacing for intermediate links are the same as short links, which accompany with the increasing stiffeners spacing, the inelastic rotation is gradually decreased. In addition, the inelastic rotations will also be decreased in large amplitude when the stiffeners with varying spacing place on one side, and the hysteresis curves with stiffeners placing on side(s) also have some differences, as shown in Fig. (9b). Comparison of the results shows that stiffeners spacing is recommended to satisfy the design spacing, and stiffeners are advisable to be placed on two sides due to the unstable performance.

Long links. The length and length ratio $\rho$ of all the long links are $2400 \mathrm{~mm}$ and 2.88 , respectively, and the design rotation is 0.02 . From Fig. $(\mathbf{8 c})$, all the stiffeners spacing are designed to satisfy the requirement by the provision, and the inelastic rotations of all the model links can reach the design rotation including a stiffener spacing with $300 \mathrm{~mm}$ from each end. Stiffeners place on two sides will increase the inelastic rotations and have a certain effect on the hysteresis curves, but these effects are not important for the overall performance of these links, as shown in Fig. (9c), only formed in the strength degradation segment. Results of analyzing the long links show that the stiffeners spacing are supposed to satisfy the design spacing, and stiffeners place on one or two sides are fine.

\subsection{Stiffener Thickness}

Links with all types of length ratio based on H400x250x10x15 are designed to analyze the effect of stiffener thickness. All of the section properties, Length, and stiffener spacing for links with varying stiffeners thickness are shown in Table 2.

Results show stiffeners thickness have a minor effect on the behaviors of the analyzing links models, and the inelastic rotations remain constant and can satisfy the design rotations by changing the stiffeners thickness, as shown in Fig. (10). Moreover, different stiffeners thicknesses don't affect the failure modes significantly. The stiffeners thickness of all types of links is only required to satisfy the provision, more than $0.75 t_{w}$ and $10 \mathrm{~mm}$.

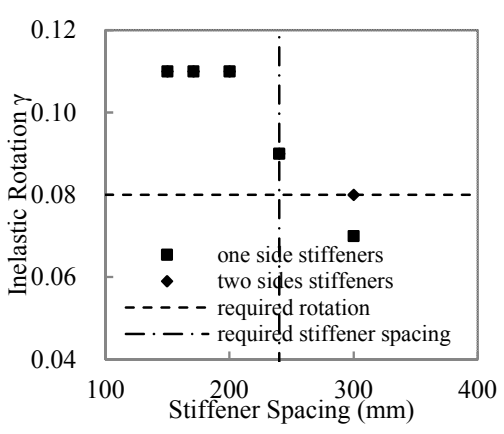

(a) short links

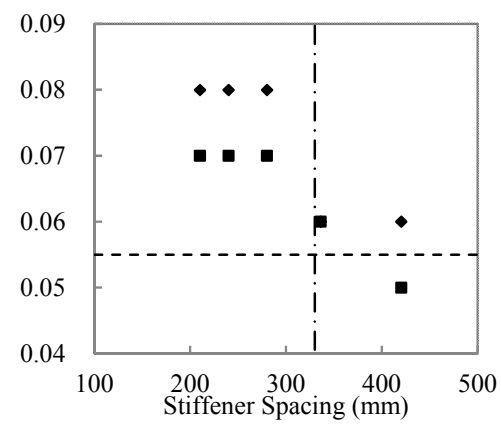

(b) intermediate links

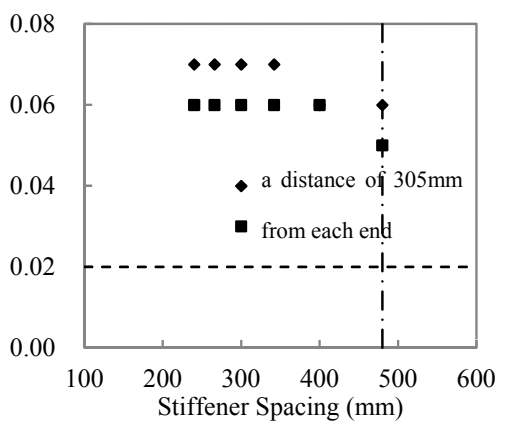

(c) long links

Fig. (8) Inelastic rotation versus stiffeners spacing and placing for model links.

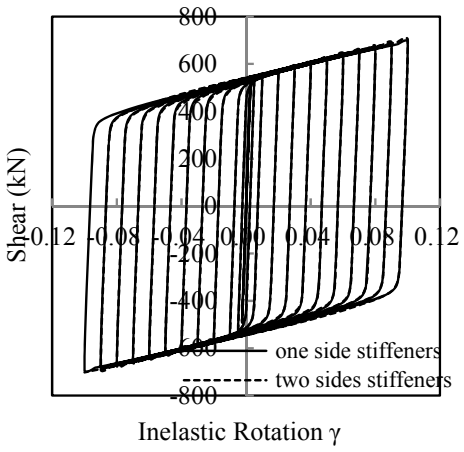

(a) short links

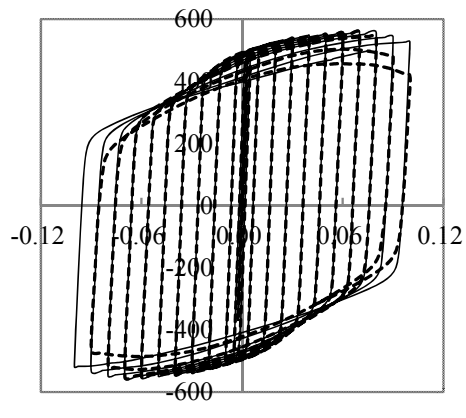

Inelastic Rotation $\gamma$

(b) intermediate links

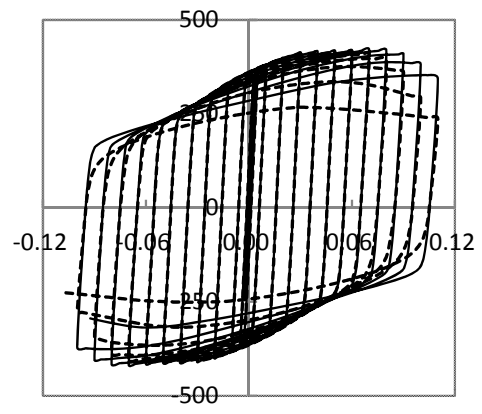

Inelastic Rotation $\gamma$

(c) long links

Fig. (9). Hysteresis curves of one side and two sides stiffeners for model links. 
Table 2. Section properties for links with varying stiffeners thickness

\begin{tabular}{|c|c|c|c|c|}
\hline Section & Type & Length & Length ratio, & Stiffener spacing \\
\hline \hline $400 \times 250 \times 10 \times 15$ & short & $1200 \mathrm{~mm}$ & 1.44 & $6 @ 200$ \\
\hline $400 \times 250 \times 10 \times 15$ & intermediate & $1680 \mathrm{~mm}$ & 2.02 & $7 @ 240$ \\
\hline $400 \times 250 \times 10 \times 15$ & long & $2400 \mathrm{~mm}$ & 2.88 & $8,6,8,10,12 \mathrm{~mm}$ ( 300 \\
\hline
\end{tabular}

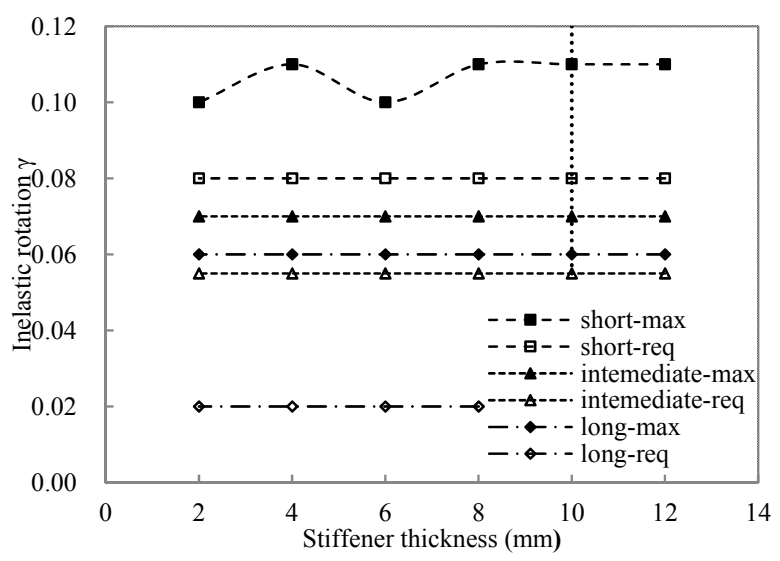

Fig. (10). Inelastic rotation versus stiffeners thickness for model links.

\subsection{Axial Force}

The effect of axial forces on the performance of links in K-type EBFs can be neglected [17]. However, axial forces are large enough to affect the behavior of links in D-type or V-type EBFs, and links with different length may be sensitive to the axial forces. Popov [18] also indicated that it is necessary to consider the effect of axial forces.

Thirty-six links models with varying length that based on H400x250x10x15 are conducted to investigate the effect of axial forces during cyclic loading. All of the section properties, length and stiffener spacing for links are shown in Table 3, and the axial forces $N$ include $0,0.05 f A, 0.10 f A \cdots$ $0.30 f A, f=$ yield stress, $A=$ section area.

All types of links are studied by finite element analysis under axial forces and cyclic loading, and the inelastic rotation versus axial forces for model links is shown in Fig. (11). The $\mathrm{X}$-coordinate is the increasing axial forces (=influencing factor of axial forces $\times f A$ ), Y-coordinate is maximum and design inelastic rotations. The four dash lines are the required inelastic rotations by the provision, such as the line with empty squares indicates the designed rotation is 0.08 for specimen 1 and specimen 2 ; the lines with filled and empty triangles are 0.075 and 0.055 for specimen 3 and 4; the line with empty rhomboids is 0.02 for specimen 5 and 6 . From Fig. (11), the axial forces have no significant influence on the behavior of model links while $N$ is less than $0.05 f A$, but the inelastic rotations and failure modes will be gradually reduced as the axial forces increase and exceed $0.05 f A$.

Specimen 1 and 2 are short links and the length ratios are 1.2 and 1.44. The axial force changed from $0.05 f A$ to $0.15 f A$ has no effect on the inelastic rotations for specimen 1 , and the inelastic rotation of specimen 2 has a minor decrease. As the axial forces increase to $0.30 \mathrm{fA}$, the inelastic rotation of specimen 1 can reach the design rotation, but specimen 2 cannot satisfy the specified value. Axial forces will have a highly influence on the inelastic rotations as the length of short links increases. The mises stresses (unit: Mpa) for

Table 3. Test section properties for links with different axial forces

$\left.\begin{array}{|c|c|c|c|c|c|c|}\hline \text { Section } & \text { Specimen } & \text { Type } & \text { Length } & \text { Length ratio, } \rho & \text { Stiffener spacing } & \text { Axial force N } \\ \hline \hline 400 \times 250 \times 10 \times 15 & 1 & \text { short } & 1000 \mathrm{~mm} & 1.20 & 5 @ 200 \\ \hline 400 \times 250 \times 10 \times 15 & 2 & \text { short } & 1200 \mathrm{~mm} & 1.44 & 6 @ 200 \\ \hline 400 \times 250 \times 10 \times 15 & 3 & \text { intermediate } & 1400 \mathrm{~mm} & 1.68 & 7 @ 200 & 7 \text { All specimens are applied } \\ 0,0.05 \mathrm{fA}, 0.1 \mathrm{fA}, 0.15 \mathrm{fA}, \\ 0.2 \mathrm{fA}, 0.25 \mathrm{fA}, 0.3 \mathrm{fA} .\end{array}\right)$




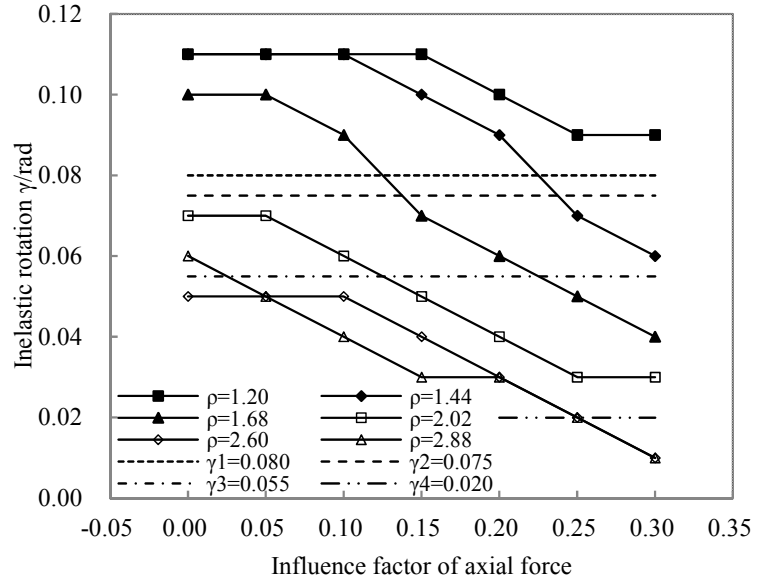

Fig. (11). Inelastic rotation versus axial forces for model links.

specimen 2 under different axial forces are shown in Fig. (12). Maximum stresses with axial forces of $0.05 \mathrm{fA}$ and $0.15 \mathrm{fA}$ are both on the webs and the failure modes have a similar agreement. So the performance of short links is stable when the axial force is less than $0.15 f A$.

Specimen 3 and 4 are intermediate links and the length ratios are 1.68 and 2.02. As the axial forces change from $0.05 f A$ to $0.15 f A$, the inelastic rotations for specimen 3 decreases from 0.1 to 0.07 that cannot reach the design rotation 0.075 , and the behavior of the specimen 4 is the same as specimen 3. Moreover, both rotations will further decrease by increasing the axial force to $0.30 \mathrm{fA}$. The mises stresses for specimen 4 under different axial forces are shown in Fig. (13). The intermediate links with the increasing axial forces from $0.05 f A$ to $0.15 f A$ are more susceptible to strength deterioration due to flange and web local buckling. So the effect of axial forces always cannot be neglected.

Specimen 5 and 6 are long links and the length ratios are 2.61 and 2.88. The inelastic rotations for specimen 5 decrease from 0.05 to 0.04 and specimen 6 changes from 0.06 to 0.03 while the axial forces are changed from $0.05 f A$ to $0.15 f A$, both specimens can reach the requirement by the provision. Moreover, both rotations will continue to decrease and fail to satisfy the design rotation as the axial force increase to $0.3 f A$. The mises stresses for specimen 6 under different axial forces are shown in Fig. (14). The local buckling of flange and premature failure of web due to the increasing stress will be affected by axial forces, and failure modes are also changed. Results show that the inelastic rotations of long links are susceptible to axial forces, it is suggested to consider this effect in spite of the inelastic rotations can meet the design value.

Results show that the effect of axial force cannot be neglected for short links unless axial force is less than $0.15 f A$, but the behaviors of intermediate and long links are unstable and should be considered under any condition, because they are susceptible to the axial forces. Comparison of the results shows that links with unignorable axial forces
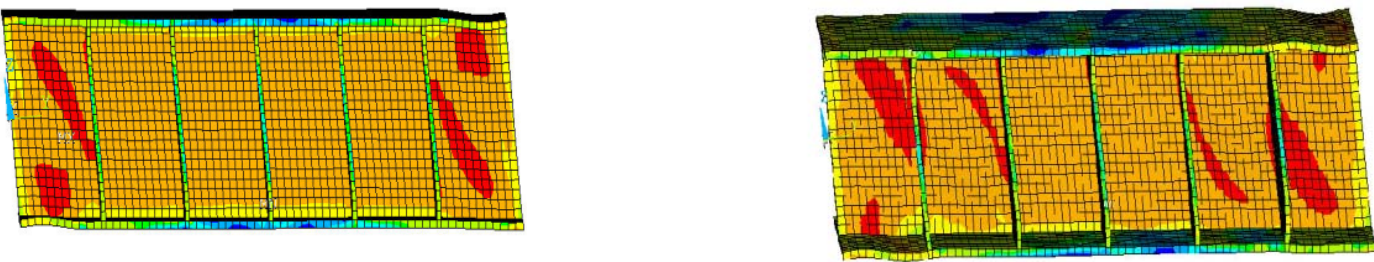

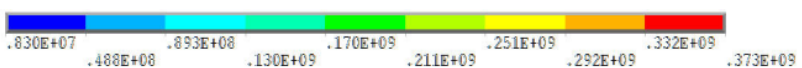

(a) $N=0.05 \mathrm{fA}$

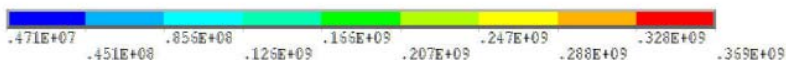

(b) $N=0.15 f A$ (Mises stress unit: $\mathrm{MPa}$ )

Fig. (12). Mises stress for specimen 2 (short link) under different axial forces.
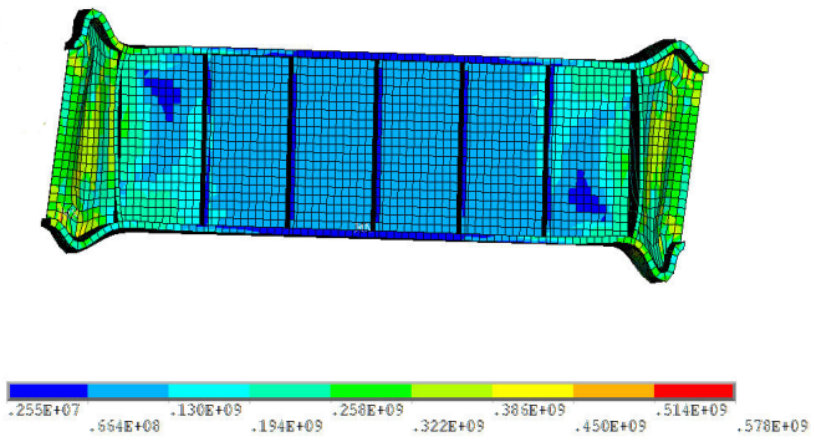

(a) $N=0.05 f A$
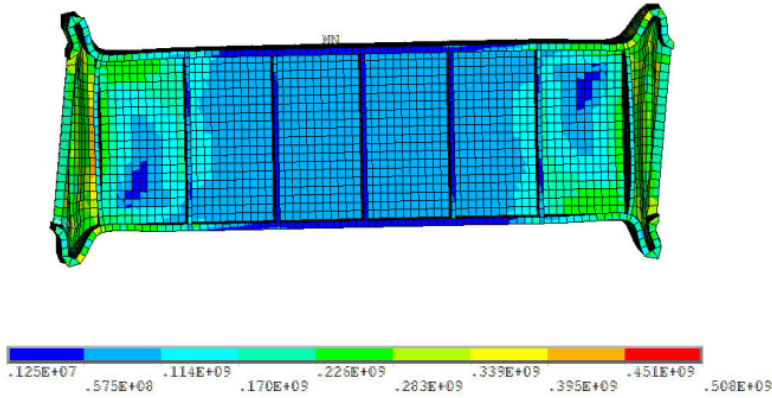

(b) $N=0.15 f A$ (Mises stress unit: $\mathrm{MPa}$ )

Fig. (13). Mises stress for specimen 4 (intermediate link) under different axial forces. 

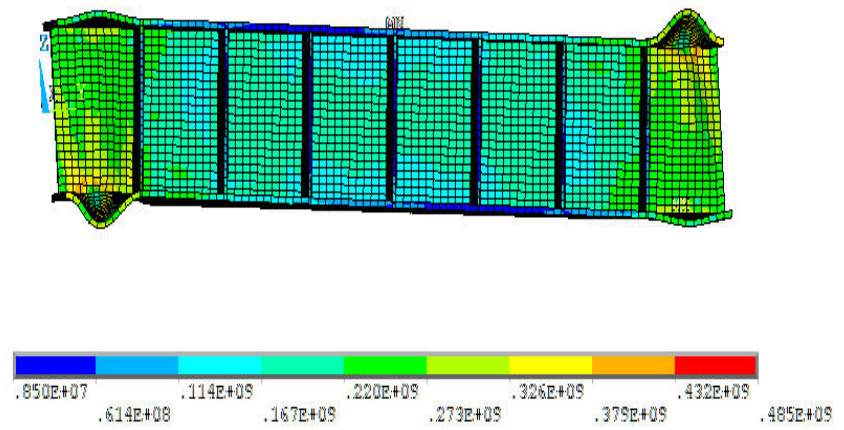

(a) $N=0.05 f A$
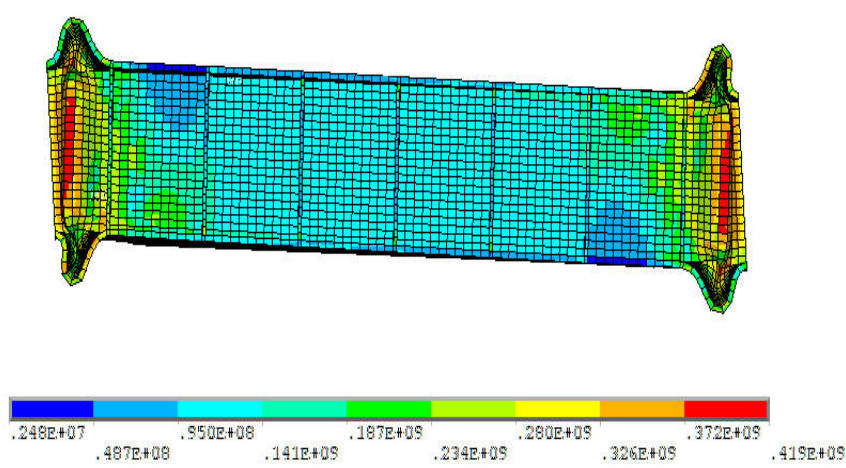

(b) $N=0.15 f A$ (Mises stress unit: $\mathrm{MPa}$ )

Fig. (14). Mises stress for specimen 6 (long link) under different axial forces.

are suggested to use short links and decrease the link length as far as possible, so the maximum inelastic rotation and stable energy dissipation can be obtained.

\section{OPTIMUM DESIGN METHOD FOR LINKS}

By means of studying the main influencing factors for links with varying length ratio, an optimum design method for links is developed according to the following steps:

(1) Selection of link sections. The first members to be sized in EBFs are links, and the link shear force in each story can be derived by the accumulated story shear force, so the web depth and thickness of link can be determined by the shear forces and web slenderness. The flange width and thickness can be obtained by considering the effect of flange width-thickness ratio, which can be relaxed to $10 \sqrt{235 / f y}$ for short and long links and be limited to $8 \sqrt{235 / f y}$ for intermediate links. Then the link sections can be selected.

(2) Stiffeners spacing. Stiffeners spacing of all types of links should only satisfy the provision.

(3) Stiffeners place on side(s). Stiffeners of short and long links can only place on one side, but stiffeners of intermediate links are advisable to place on two sides.

(4) Stiffeners thickness. The stiffeners thicknesses of all types of links are only required to satisfy the provision, more than $0.75 t_{w}$ and $10 \mathrm{~mm}$.

Moreover, short links will be the most proper links when the axial force should be considered, and the length ratio from 1.3 to 1.5 would be the best choice.

\section{CONCLUSION}

Effective finite element investigation of 156 links is conducted to comprehensively study the main influencing factors and global behavior of all types of links with Ishaped cross-section during cyclic loading. The conclusions are as follows:

(1) The flange width-thickness ratio can be relaxed to $10 \sqrt{235 / \text { fy }}$ for short and long links, and the flange width- thickness ratio of intermediate links is always limited to $8 \sqrt{235 / \mathrm{fy}}$.

(2) Analysis results indicate that stiffeners spacing of links with varying length ratio have no significant effect on the inelastic rotations, so it is suggested to satisfy the design spacing. Stiffeners of short and long links can only be placed on one side, but stiffeners of intermediate links are advisable to be placed on two sides due to the unstable behavior.

(3) Different stiffeners thicknesses do not affect the failure modes and inelastic rotations significantly. Therefore, more than $0.75 t_{w}$ and $10 \mathrm{~mm}$ that is considered the structural factor by the provision is suggested.

(4) The effect of axial force of short links should only be considered when the axial force is more than $0.15 f A$, but the behaviors of intermediate and long links are unstable and susceptible to the axial forces, so it cannot be neglected under any condition. Furthermore, the ductility and strength of short links are better than the other links, it is suggested to use short links and decrease the link length when axial force should be considered, then the maximum inelastic rotation and stable energy dissipation can be obtained.

(5) An optimum design method is proposed by analyzing the main influencing factors for all types of links.

\section{CONFLICT OF INTEREST}

The authors confirm that this article content has no conflicts of interest

\section{ACKNOWLEDGEMENTS}

The authors gratefully acknowledge the research grant provided by the National Nature Science Foundation of China (No. 51178192; 51378219; 51378009), State Key Laboratory of Subtropical Building Science Foundation, South China University of Technology (No. 2012ZA05).

\section{REFERENCES}

[1] K. Kasai, and E. P. Popov, "General Behavior of WF Steel Shear Link Beams", J. Struct. Eng., ASCE, vol.112, pp. 362-382, July 1986. 
[2] K. Kasai, and E. P. Popov, "Cyclic Web Buckling Control of Shear Link Beams", J. Struct. Eng., ASCE, vol. 112, pp. 505-523, August 1986.

[3] J. Rozon, S. Koboevic, and R. Tremblay, "Study of Global Behavior of Eccentrically Braced Frames in Response to Seismic Loads", The 14th World Conference on Earthquake Engineering, Beijing, October 2008.

[4] Y.J. Shi, J. Xiong, and Y. Q. Wang, "Experimental studies on seismic performance of multi-storey steel frame with eccentric brace", J. Build Struct., vol.31, pp. 29-34, February 2010. (in Chinese)

[5] T. A. Sabol, and D.E. Nishi, "Application of performance-based design to an eccentrically braced frame structure", Struct. Design Tall Spec. Build, vol.20, pp.76-84, December 2011.

[6] AISC. "Seismic Provisions for Structural Steel Buildings", American Institute of Steel Construction: Chicago, Illinois, 2002.

[7] P. W. Richards, and C. M. Uang, "Effect of flange width-thickness ratio on eccentrically braced frames link cyclic rotation capacity", J. Struct. Eng., ASCE, vol.131, pp.1546-1552, October 2005.

[8] P. W. Richards, and C. M. Uang, "Testing protocol for short links in eccentrically braced frames", J. Struct. Eng., ASCE, vol.132, pp.1183-1191,August 2006.

[9] T. Okazaki, and M. D. Engelhardt, "Cyclic loading behavior of EBF links constructed of ASTM A992 steel", J. Construct. Steel Res., vol.63, pp.751-765, June 2007.
[10] P. P. Rossi, and A. Lombardo, "Influence of the link overstrength factor on the seismic behavior of eccentrically braced frames", $J$. Construct. Steel Res., vol.63, pp.1529-1545, November 2007.

[11] J. W. Berman, and M. Bruneau, "Experimental and analytical investigation of tubular links for eccentrically braced frames", Eng. Struct., vol.29, pp.1929- 1938, August 2007.

[12] Daneshmand, and B. H. Hashemi, "Performance of intermediate and long links in eccentrically braced frames", J. Construct. Steel Res., vol.70, pp. 167-176, January 2012.

[13] Mohebkhah, and B.Chegeni. "Overstrength and rotation capacity for EBF links made of European IPE sections." Thin-Walled Structures, vol.74, pp. 255-260, February 2014.

[14] GB50010-2010, Code for seismic design of building, China Architecture \& Building Press: Beijing, 2010, pp.98-110. (in Chinese)

[15] X.M. Wang. "ANSYS numerical analysis of engineering structures". China Communications Press: 2007. (in Chinese)

[16] B.S. Guo, X.Y. Zhuang, and Y.M. Yan, "Effects of the link's configu- ration on load-bearing characteristic of $\mathrm{K}$ shape eccentrically braced steel frames", Xi'an Univ. of Arch. Tech., vol. 39, pp. 155-160, April 2007. (in Chinese)

[17] Ghobarah, and T. Ramadan, "Effect of axial forces on the performance of links in eccentrically braced frames", Eng. Struct., vol.12, pp. 106-113, February 1990.

[18] E.P. Popov, and M. D. Engelhardt, "Seismic eccentrically braced frames", J. Construct. Steel Res., vol.10, pp.321-354, October 1988.

(C) Hu and Wang; Licensee Bentham Open.

This is an open access article licensed under the terms of the Creative Commons Attribution Non-Commercial License (http://creativecommons.org/licenses/by-nc/3.0/) which permits unrestricted, non-commercial use, distribution and reproduction in any medium, provided the work is properly cited. 